\title{
Two-Dimensional Sparse Arrays with Hole-Free Coarray and Reduced Mutual Coupling
}

\author{
Chun-Lin Liu and P. P. Vaidyanathan \\ Dept. of Electrical Engineering, 136-93 \\ California Institute of Technology, Pasadena, CA 91125, USA \\ cl.liu@ caltech.edu, ppvnath@ systems.caltech.edu
}

\begin{abstract}
Two-dimensional sparse arrays with hole-free difference coarrays, like billboard arrays and open box arrays, can identify $O\left(N^{2}\right)$ uncorrelated source directions (DOA) using $N$ sensors. These arrays contain some dense ULA segments, leading to many sensor pairs separated by $\lambda / 2$. The DOA estimation performance often suffers degradation due to mutual coupling between such closely-spaced sensor pairs. This paper introduces a new 2D array called the half open box array. For a given $N$, this array has the same hole-free coarray as an open box array. At the same time, the number of sensor pairs with small separation is significantly reduced.
\end{abstract}

Index Terms-Planar sparse arrays, open box arrays, partially open box arrays. half open box arrays, mutual coupling, DOA estimation.

\section{INTRODUCTION}

Planar arrays find useful applications in beamforming, radar, imaging, and commuincations [1]-[3]. They can jointly estimate the azimuth and elevation of sources [1]. Some wellknown planar array geometries include uniform rectangular arrays (URA), uniform circular arrays (UCA), and hexangonal arrays, in which elements are placed uniformly on regular contours [1]. However, these array configurations usually suffer from significant mutual coupling, resulting in considerable interferences between sensor outputs [4], [5].

It is well-known that large sensor separations help to reduce the mutual coupling effect [4], [5]. Hence, linear sparse arrays, in which the number of sensor pairs with small separations is much smaller than uniform linear arrays (ULA), are more robust to mutual coupling [6]. Examples of linear sparse arrays include minimum redundancy arrays (MRA) [7], nested arrays [8], coprime arrays [9], super nested arrays [6], [10], and other generalizations [11]. All these sparse arrays can resolve $O\left(N^{2}\right)$ uncorrelated sources given $O(N)$ sensors, whereas ULAs identify at most $N-1$ sources with $N$ elements [1], [6][9], [11]. Among these, super nested arrays and coprime arrays are significantly robust to mutual coupling effects because they have very few sensor pairs with small separations. Super nested arrays have an additional advantage over coprime arrays because the coarrays are filled (hole-free). Unlike MRAs which share the hole-free property, the sensor locations in a super nested array also have a simple closed form.

For planar arrays, it is also desirable to have closed-form sensor locations, large difference coarrays, and less mutual coupling, like super nested arrays. However, such planar arrays are not explored in literature. Some existing designs enjoy closed-form sensor locations with hole-free coarray, including billboard arrays, 2D nested arrays, and open box arrays [12][14]. Hence, these planar sparse arrays can also distinguish

\footnotetext{
${ }^{1}$ This work was supported in parts by the ONR grant N00014-15-1-2118, and the California Institute of Technology.
}

more sources than sensors [15]. Nevertheless, none of them takes the mutual coupling issue into account.

In this paper, starting from open box arrays, we will develop a novel family of planar arrays, called partially open box arrays. By redistributing sensors properly, partially open box arrays are guaranteed to possess hole-free coarrays with enhanced degrees of freedom, which makes it possible to detect more sources than sensors. Next, we will present half open box arrays, which inherit most of the good properties of partially open box arrays. Moreover, the number of sensor pairs with unit horizontal spacing $(\lambda / 2)$ in half open box arrays, is as small as 2 , indicating that mutual coupling decreases significantly. These properties will be given in depth later.

This paper is outlined as follows. Section II reviews the data model and several well-known planar arrays, like URA, billboard arrays, 2D nested arrays, and open box arrays. In Section III, open box arrays are generalized into partially open box arrays and half open box arrays. Section IV demonstrates the superior performance for the proposed arrays in the presence of mutual coupling while Section V concludes this paper.

\section{PRELIMINARIES}

\section{A. The Data Model}

Suppose $D$ uncorrelated sources impinge on a planar array, whose sensors are located at $\mathbf{n} d$. Here $\mathbf{n}=\left(n_{x}, n_{y}\right) \in \mathbb{Z}^{2}$ is an integer-valued vector and $d=\lambda / 2$ is the minimum separation between sensors. The sensor locations $\mathbf{n}$ form a set $\mathbb{S}$. The $i$ th source has complex amplitude $A_{i} \in \mathbb{C}$, azimuth $\phi_{i} \in[0,2 \pi]$, and elevation $\theta_{i} \in[0, \pi]$. If mutual coupling is absent, the sensor output on $\mathbb{S}$ can be modeled as

$$
\mathbf{x}_{\mathbb{S}}=\sum_{i=1}^{D} A_{i} \mathbf{v}_{\mathbb{S}}\left(\bar{\theta}_{i}, \bar{\phi}_{i}\right)+\mathbf{n}_{\mathbb{S}}
$$

where $\bar{\theta}_{i}=(d / \lambda) \sin \theta_{i} \cos \phi_{i}$ and $\bar{\phi}_{i}=(d / \lambda) \sin \theta_{i} \sin \phi_{i}$ are the normalized DOA. The element of the steering vector $\mathbf{v}_{\mathbb{S}}\left(\bar{\theta}_{i}, \bar{\phi}_{i}\right)$ corresponding to the sensor at $\left(n_{x}, n_{y}\right) \in \mathbb{S}$ is $e^{j 2 \pi\left(\bar{\theta}_{i} n_{x}+\bar{\phi}_{i} n_{y}\right)}$. Signals and noise are assumed to be zero-mean and uncorrelated. That is, $\mathbb{E}\left[A_{i}\right]=0, \mathbb{E}\left[\mathbf{n}_{\mathbb{S}}\right]=$ $\mathbf{0}, \mathbb{E}\left[A_{i} A_{j}^{*}\right]=\sigma_{i}^{2} \delta_{i, j}, \mathbb{E}\left[\mathbf{n}_{\mathbb{S}} \mathbf{n}_{\mathbb{S}}^{H}\right]=\sigma^{2} \mathbf{I}, \mathbb{E}\left[A_{i} \mathbf{n}_{S}^{H}\right]=\mathbf{0}$, where $\sigma_{i}^{2}$ and $\sigma^{2}$ are the $i$ th source power and the noise power, respectively. $\delta_{p, q}$ is the Kronecker delta.

For uncorrelated sources, the covariance matrix of $\mathbf{x}_{\mathbb{S}}$ can be expressed as

$$
\mathbf{R}_{\mathbb{S}}=\mathbb{E}\left[\mathbf{x}_{\mathbb{S}} \mathbf{x}_{\mathbb{S}}^{H}\right]=\sum_{i=1}^{D} \sigma_{i}^{2} \mathbf{v}_{\mathbb{S}}\left(\bar{\theta}_{i}, \bar{\phi}_{i}\right) \mathbf{v}_{\mathbb{S}}^{H}\left(\bar{\theta}_{i}, \bar{\phi}_{i}\right)+\sigma^{2} \mathbf{I} .
$$


Vectorizing (2) and removing duplicated entries yield the signal on the difference coarray:

$$
\mathbf{x}_{\mathbb{D}}=\sum_{i=1}^{D} \sigma_{i}^{2} \mathbf{v}_{\mathbb{D}}\left(\bar{\theta}_{i}, \bar{\phi}_{i}\right)+\sigma^{2} \mathbf{e}_{0},
$$

where $\mathbf{e}_{0}$ is a column vector with $\left\langle\mathbf{e}_{0}\right\rangle_{\left(n_{x}, n_{y}\right)}=\delta_{n_{x}, 0} \delta_{n_{y}, 0}$. The bracket notation $\left\langle\mathbf{x}_{\mathbb{S}}\right\rangle_{\mathbf{n}}$ denotes the value of the signal at the support location $\mathbf{n} \in \mathbb{S}$. For instance, if $\mathbb{S}=$ $\{(0,0),(1,0),(0,1)\}$ and $\mathbf{x}_{\mathbb{S}}=[4,5,6]^{T}$, then $\left\langle\mathbf{x}_{\mathbb{S}}\right\rangle_{(0,0)}=4$, $\left\langle\mathbf{x}_{\mathbb{S}}\right\rangle_{(1,0)}=5$, and $\left\langle\mathbf{x}_{\mathbb{S}}\right\rangle_{(0,1)}=6[16] . \mathbb{D}$ is the difference coarray, which is defined as

Definition 1 (Difference coarray). For a planar array specified by $\mathbb{S}$, its difference coarray $\mathbb{D}$ is defined as the differences between sensor locations:

$$
\mathbb{D}=\left\{\mathbf{n}_{1}-\mathbf{n}_{2} \mid \mathbf{n}_{1}, \mathbf{n}_{2} \in \mathbb{S}\right\}
$$

For example, if $\mathbb{S}$ consists of $(0,0),(1,0),(2,0),(0,1)$, $(2,1),(0,2),(2,2)$, then the difference coarray $\mathbb{D}$ is composed of integer vectors $\left(m_{x}, m_{y}\right)$ such that $-2 \leq m_{x}, m_{y} \leq 2$. The uniform rectangular part of $\mathbb{D}$ is denoted by $\mathbb{U}$. In this example, $\mathbb{D}=\mathbb{U}$, and such array is said to have a hole-free coarray.

The set $\mathbb{U}$ influences the estimation capability of the array $\mathbb{S}$. It is possible to resolve source DOAs using 2D spatial smoothing MUSIC based on the signals on $\mathbb{U}[15]$. Furthermore, the number of identifiable uncorrelated sources is of the order of the size of $\mathbb{U}$, under realistic assumptions [17]. As a result, for appropriate sparse arrays with $N$ physical sensors, the sizes of $\mathbb{D}$ and $\mathbb{U}$ are both $O\left(N^{2}\right)$, indicating that we can identify more sources than sensors.

If mutual coupling is present, the data model (1) becomes

$$
\mathbf{x}_{\mathbb{S}}=\sum_{i=1}^{D} A_{i} \mathbf{C v}_{\mathbb{S}}\left(\bar{\theta}_{i}, \bar{\phi}_{i}\right)+\mathbf{n}_{\mathbb{S}}
$$

where $\mathbf{C}$ is the mutual coupling matrix. The entries of $\mathbf{C}$ can be written as [4]

$$
\langle\mathbf{C}\rangle_{\mathbf{n}_{1}, \mathbf{n}_{2}}= \begin{cases}c\left(\left\|\mathbf{n}_{1}-\mathbf{n}_{2}\right\|_{2}\right), & \text { if }\left\|\mathbf{n}_{1}-\mathbf{n}_{2}\right\|_{2} \leq B, \\ 0 & \text { otherwise, }\end{cases}
$$

where $\mathbf{n}_{1}, \mathbf{n}_{2} \in \mathbb{S}$ denote the sensor locations. Here $\|\cdot\|_{2}$ is the $\ell_{2}$-norm of a vector and $c(\cdot)$ are the mutual coupling coefficients. It is assumed that $c(0)=1$ and $|c(k) / c(\ell)|=\ell / k$ [4], implying that the arrays with larger sensor separations, like sparse arrays, tend to reduce mutual coupling. To quantify mutual coupling, we first define the weight function:

Definition 2 (Weight function). Let a planar array be specified by $\mathbb{S}$, and let its difference coarray be $\mathbb{D}$. The weight function $w(\mathbf{m})$ is the number of pairs with separation $\mathbf{m} \in \mathbb{D}$, i.e.,

$$
w(\mathbf{m})=\left|\left\{\left(\mathbf{n}_{1}, \mathbf{n}_{2}\right) \in \mathbb{S}^{2} \mid \mathbf{n}_{1}-\mathbf{n}_{2}=\mathbf{m}\right\}\right|
$$

We will use $w(\mathbf{m})$ and $w\left(m_{x}, m_{y}\right)$ interchangeably if $\mathbf{m}=$ $\left(m_{x}, m_{y}\right)$. It was shown in [6] that smaller weight functions at small sensor separations reduce the effect of mutual coupling significantly.

\section{B. Known Closed-Form Planar Sparse Arrays}

In this subsection, we will review some known planar arrays on rectangular grids with regular geometries, in Fig. 1.

The URA places $N_{x} N_{y}$ sensors on an $N_{y}$-by- $N_{x}$ rectangular grid, as demonstrated in Fig. 1(a) for 36 sensors. The billboard array [12] consists of three ULA on a square aperture $\left(N_{x}=N_{y}\right)$ and the total number of sensors is $3\left(N_{x}-1\right)$. The 2D nested array [14] is depicted in Fig. 1(c). In this example, this array is the cross product of two identical 1D nested arrays with $N_{1}=N_{2}=3$ (notation as in [8]) and the number of sensors is $\left(N_{1}+N_{2}\right)^{2}$. Finally, the open box array [13] assigns $N_{x}+2 N_{y}-2$ sensors on the boundaries of a rectangular aperture, which is formally defined as

Definition 3 (Open box arrays). Let $N_{x}$ and $N_{y}$ be positive integers satisfying $N_{x} \geq N_{y}$. An open box array is characterized by a integer set $\mathbb{S}_{\mathrm{OBA}}$, defined by

$$
\begin{aligned}
\mathbb{S}_{\text {OBA }}= & \left\{(0,0),\left(N_{x}-1,0\right),\left(0, N_{y}-1\right),\left(N_{x}-1, N_{y}-1\right)\right\} \\
& \cup \mathbb{G}_{1} \cup \mathbb{H}_{1} \cup \mathbb{H}_{2},
\end{aligned}
$$

where $\mathbb{G}_{1}=\left\{\left(n_{x}, 0\right) \mid n_{x} \in \mathrm{g}_{1}\right\}, \mathbb{H}_{1}=\left\{\left(0, n_{y}\right) \mid n_{y} \in \mathbb{h}_{1}\right\}$, and $\mathbb{H}_{2}=\left\{\left(N_{x}-1, n_{y}\right) \mid n_{y} \in \mathbb{h}_{2}\right\}$. Here $\mathrm{g}_{1}=\left\{1,2, \ldots, N_{x}-2\right\}$ and $\mathrm{h}_{1}=\mathbb{h}_{2}=\left\{1,2, \ldots, N_{y}-2\right\}$.

Fig. 1(d) marks the sets $\mathbb{G}_{1}, \mathbb{H}_{1}$, and $\mathbb{H}_{2}$ in rectangles on the bottom, on the left, and on the right, respectively.

All of the arrays in Fig. 1 have 36 physical sensors and hole-free coarrays $(\mathbb{D}=\mathbb{U})$. However, the sizes of difference coarrays are different. The largest $|\mathbb{D}|$ is exhibited by the open box array (665), followed by the billboard array (625), the 2D nested array (529), and finally the URA (121). Larger $|\mathbb{D}|$ offers better spatial resolution and more resolvable uncorrelated sources, so that the open box array is preferred in Fig. 1.

Next, we will consider the weight functions with small separations, such as $w(1,0), w(0,1), w(1,1)$, and $w(1,-1)$, as listed in Fig. 1. Notice that for the arrays mentioned above these weights are not small. For instance, the open box array has $w(1,0)=17$ and $w(0,1)=18$, due to the dense ULA on the boundaries. It is desirable to reduce $w(1,0), w(0,1)$, $w(1,1)$, and $w(1,-1)$ simultaneously, so that mutual coupling can be mitigated.

\section{Generalization OF Open Box ARrays}

In this section, we will develop generalizations of open box arrays. The reason why we start with open box arrays is that, based on Fig. 1, they have the largest aperture for the same number of sensors, leading to the best spatial resolution.

\section{A. Partially Open Box Arrays}

The main idea of partially open box arrays is to redistribute the elements in the dense ULA, so that the weight functions for small separations decrease. In this paper, we focus on the set $\mathbb{G}_{1} \cup\left\{(0,0),\left(N_{x}-1,0\right)\right\}$, i.e., the $N_{x}$ sensors on the bottom of Fig. 1(d). These sensors contribute to the weight function $w(1,0)$. If we can relocate some of these sensors, it is possible to reduce $w(1,0)$.

However, if we move these sensors arbitrarily, the difference coarray would no longer be hole-free so that the spatial resolution is degraded. To keep the difference coarray intact, we consider the following notations: Let $\mathbb{S}_{\text {OBA }}$ be an open box array with sizes $N_{x}$ and $N_{y}$, as in Definition 3, and let $\mathbb{D}_{\text {OBA }}$ be the difference coarray. Assume we select $P$ distinct sensors, located at $\left(n_{p}, 0\right) \in \mathbb{S}_{\mathrm{OBA}}$ for $p=1,2, \ldots, P$ and $P<N_{x}$, These sensors are relocated to $P$ distinct locations, 


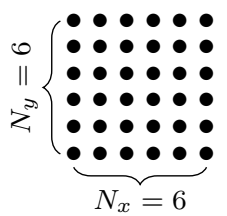

(a) the URA

Aperture: $5 \times 5=25$ $w(1,0)=30, w(0,1)=30$, $w(1,1)=25, w(1,-1)=25$.

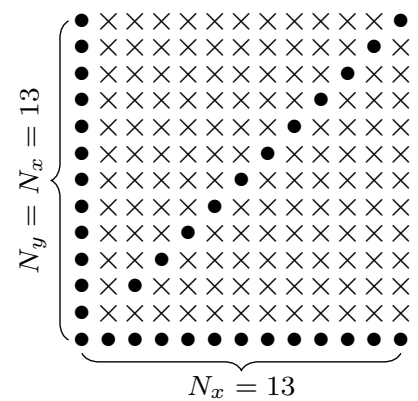

(b) the billboard array Aperture: $12 \times 12=144$ $w(1,0)=12, w(0,1)=12$, $w(1,1)=10, w(1,-1)=1$.

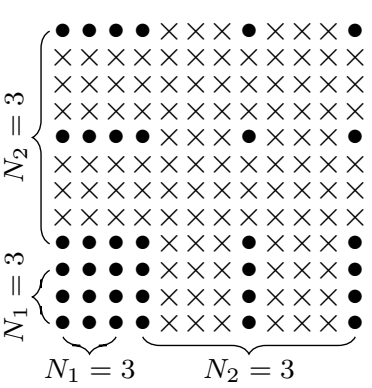

(c) the 2D nested array Aperture: $11 \times 11=121$ $w(1,0)=18, w(0,1)=18$, $w(1,1)=9, w(1,-1)=9$.

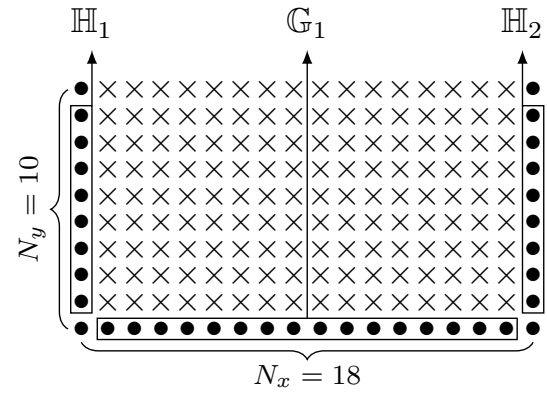

(d) the open box array Aperture: $9 \times 17=153$ $w(1,0)=17, w(0,1)=18$, $w(1,1)=1, w(1,-1)=1$.

Fig. 1. Examples of planar arrays with $N=36$ elements. Bullets denote physical sensors and crosses represent empty space. The minimum separation between sensors is half of the wavelength of the incoming waveform.

$\left(a_{p}, b_{p}\right) \notin \mathbb{S}_{\mathrm{OBA}}$, yielding a new planar array $\mathbb{S}^{\prime}$ and its difference coarray $\mathbb{D}^{\prime}$. Then we have the following lemma:

Lemma 1. $\mathbb{D}_{\mathrm{OBA}}=\mathbb{D}^{\prime}$ only if $1 \leq a_{p} \leq N_{x}-2$ and $1 \leq$ $b_{p} \leq N_{y}-1$ for all $p=1,2, \ldots, P$, i.e., only if the new sensor locations are inside the original array aperture.

Proof. According to Definition 3, the difference coarray $\mathbb{D}$ can be expressed as

$$
\begin{aligned}
\mathbb{D}_{\mathrm{OBA}}=\{ & \left(m_{x}, m_{y}\right) \in \mathbb{Z}^{2} \mid-N_{x}+1 \leq m_{x} \leq N_{x}-1 \\
& \left.-N_{y}+1 \leq m_{y} \leq N_{y}-1\right\}
\end{aligned}
$$

The proof can be divided into four cases:

1) If $a_{p}<0$, consider the sensor pair in $\mathbb{S}^{\prime}:\left(N_{x}-1, N_{y}-1\right)$ and $\left(a_{p}, b_{p}\right)$. Their difference is $\left(N_{x}-1-a_{p}, N_{y}-1-b_{p}\right) \notin$ $\mathbb{D}_{\mathrm{OBA}}$, since the first coordinate $N_{x}-1-a_{p}>N_{x}-1$.

2) If $a_{p}>N_{x}-1$, for the sensor pair $\left(a_{p}, b_{p}\right),\left(0, N_{y}-1\right) \in$ $\mathbb{S}^{\prime}$, the difference becomes $\left(a_{p}, b_{p}-N_{y}+1\right) \notin \mathbb{D}_{\mathrm{OBA}}$ because $a_{p}>N_{x}-1$.

3) If $b_{p}<0$, we can take the sensor pair of $\left(0, N_{y}-1\right)$ and $\left(a_{p}, b_{p}\right)$. The difference is $\left(-a_{p}, N_{y}-1-b_{p}\right) \notin \mathbb{D}_{\mathrm{OBA}}$.

4) If $b_{p}>N_{y}-1$, we have the following chain of arguments. Since $P<N_{x}$, there must exist a element $\left(n^{\prime}, 0\right) \in \mathbb{S}^{\prime}$. Then the difference between $\left(a_{p}, b_{p}\right)$ and $\left(n^{\prime}, 0\right)$ is $\left(a_{p}-\right.$ $\left.n^{\prime}, b_{p}\right) \notin \mathbb{D}_{\mathrm{OBA}}$, because $b_{p}>N_{y}-1$.

These arguments show that $0 \leq a_{p} \leq N_{x}-1$ and $0 \leq b_{p} \leq$ $N_{y}-1$ are necessary for $\mathbb{D}_{\mathrm{OBA}}=\mathbb{D}^{\prime}$. Furthermore, since $\left(a_{p}, b_{p}\right) \notin \mathbb{S}_{\mathrm{OBA}}$, the necessary condition becomes $1 \leq a_{p} \leq$ $N_{x}-2$ and $1 \leq b_{p} \leq N_{y}-1$, which proves this lemma.

Lemma 2. $\mathbb{D}_{\mathrm{OBA}}=\mathbb{D}^{\prime}$ only if $(0,0) \in \mathbb{S}^{\prime}$ and $\left(N_{x}-1,0\right) \in \mathbb{S}^{\prime}$, where all notations are as stated before Lemma 1.

Proof. Assume that $\mathbb{D}_{\mathrm{OBA}}=\mathbb{D}^{\prime}$. We obtain $\left(N_{x}-1, N_{y}-1\right) \in$ $\mathbb{D}_{\text {OBA }}=\mathbb{D}^{\prime}$. Due to Lemma 1 , the only sensor pair with this separation is $\left(N_{x}-1, N_{y}-1\right)$ and $(0,0)$, implying $(0,0) \in \mathbb{S}^{\prime}$. Similar arguments applies to the sensor pair of $\left(N_{x}-1,0\right)$ and $\left(0, N_{y}-1\right)$, which proves this lemma.

Lemma 1 and 2 indicate that for the sensors located on the bottom of open box arrays, only those at $(n, 0)$, where $1 \leq$ $n \leq N_{x}-2$, can be redistributed within the original aperture. For simplicity, we assume all the new sensor locations have $y$-coordinate $N_{y}-1$, i.e., $b_{p}=N_{y}-1$ for all $p$ in Lemma 1, which leads to the definition of partially open box arrays: (a)

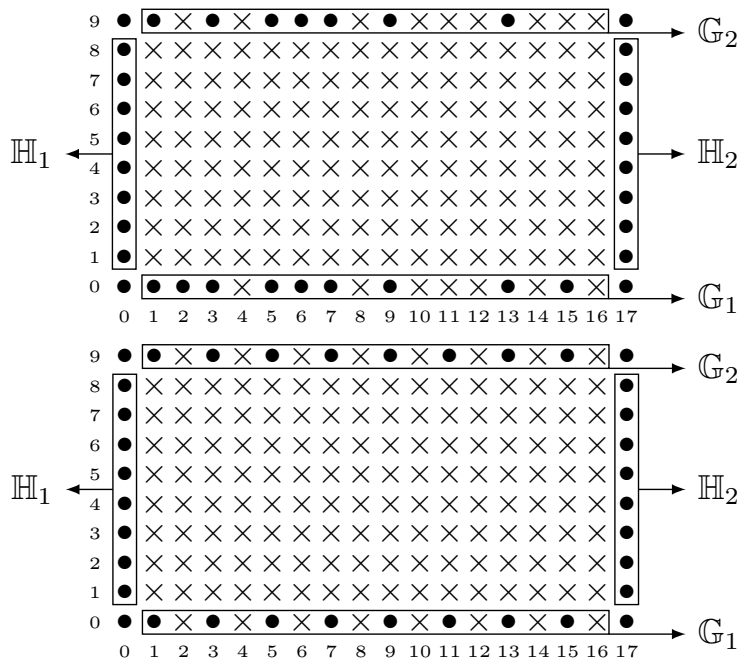

Fig. 2. Examples of partially open box arrays with $N_{x}=18$ and $N_{y}=$ 10. (a) $\mathrm{g}_{1}=\{1,2,3,5,6,7,9,13,15\}, \mathrm{g}_{2}=\{1,3,5,6,7,9,13\}$, and (b) $\mathrm{g}_{1}=\mathrm{g}_{2}=\{1,3,5,7,9,11,13,15\}$. In both cases, $\mathrm{g}_{2}$ satisfies Theorem 1 .

Definition 4 (Partially open box arrays). For two positive integers $N_{x}$ and $N_{y}$ with $N_{x} \geq N_{y}$, a partially open box array has the sensor locations defined by the integer set $\mathbb{S}_{\mathrm{POBA}}$,

$\mathbb{S}_{\text {POBA }}=\left\{(0,0),\left(N_{x}-1,0\right),\left(0, N_{y}-1\right),\left(N_{x}-1, N_{y}-1\right)\right\}$ $\cup \mathbb{G}_{1} \cup \mathbb{G}_{2} \cup \mathbb{H}_{1} \cup \mathbb{H}_{2}$,

where $\mathbb{G}_{1}=\left\{\left(n_{x}, 0\right) \mid n_{x} \in \mathbb{g}_{1}\right\}, \mathbb{G}_{2}=\left\{\left(n_{x}, N_{y}-1\right) \mid n_{x} \in\right.$ $\left.\mathrm{g}_{2}\right\}, \mathbb{H}_{1}=\left\{\left(0, n_{y}\right) \mid n_{y} \in \mathbb{h}_{1}\right\}, \mathbb{H}_{2}=\left\{\left(N_{x}-1, n_{y}\right) \mid n_{y} \in\right.$ $\left.\mathbb{h}_{2}\right\}$. Here $\mathrm{g}_{1}, \mathrm{~g}_{2}, \mathfrak{h}_{1}$, and $\mathbb{h}_{2}$ satisfy

1) $g_{1}$ and $g_{2}$ are subsets of $\left\{1,2, \ldots, N_{x}-2\right\}$.

2) $\left|g_{1}\right|+\left|g_{2}\right|=N_{x}-2$.

3) $\mathfrak{h}_{1}=\mathbb{h}_{2}=\left\{1,2, \ldots, N_{y}-2\right\}$.

To give some feelings for partially open box arrays, let us consider two examples in Fig. 2, where $N_{x}=18, N_{y}=10$ and the sets $\mathbb{G}_{1}, \mathbb{G}_{2}, \mathbb{H}_{1}$, and $\mathbb{H}_{2}$ are marked in rectangles. Fig. 2(a) illustrates a partially open box array with $\mathrm{g}_{1}=\{1,2,3,5,6,7,9,13,15\}$ and $\mathrm{g}_{2}=\{1,3,5,6,7,9,13\}$, which are subsets of $\{1,2, \ldots, 16\}$. Furthermore, $\left|g_{1}\right|+\left|g_{2}\right|=$ 
$9+7=16$ satisfies the second item in Definition 4. Fig. 2(b) also satisfies Definition 4. The missing elements (crosses) in $\mathbb{G}_{1}$ migrate to the elements (bullets) in $\mathbb{G}_{2}$.

Next, we will develop the difference coarray of partially open box arrays. The following theorem states a necessary and sufficient condition under which a open box array and a partially open box array share the same hole-free difference coarray:

Theorem 1. Consider an open box array and a partially open box array with the same $N_{x}$ and $N_{y}$, as defined in Definition 3 and 4, respectively. Then, their difference coarrays are identical if and only if $\left\{\mathrm{g}_{1}, N_{x}-1-\mathrm{g}_{2}\right\}$ is a partition of $\left\{1,2, \ldots, N_{x}-2\right\}$, i.e., if and only if 1) $\mathrm{g}_{1} \cup\left(N_{x}-1-\mathrm{g}_{2}\right)=$ $\left\{1,2, \ldots, N_{x}-2\right\}$ and 2) $\mathrm{g}_{1}$ and $N_{x}-1-\mathrm{g}_{2}$ are disjoint. Here $N_{x}-1-\mathrm{g}_{2}=\left\{N_{x}-1-g \mid g \in \mathrm{g}_{2}\right\}$.

Proof. Let $\mathbb{S}_{\mathrm{OBA}}$ and $\mathbb{S}_{\mathrm{POB}}$ be an open box array and a partially open box array, respectively. Their difference coarrays are denoted by $\mathbb{D}_{\mathrm{OBA}}$ and $\mathbb{D}_{\mathrm{POBA}}$. It is clear that $\mathbb{D}_{\mathrm{POBA}} \subseteq$ $\mathbb{D}_{\mathrm{OBA}}$, due to (6) and Lemma 1.

(Sufficiency) We will show that if $\left\{\mathrm{g}_{1}, N_{x}-1-\mathrm{g}_{2}\right\}$ is a partition of $\left\{1,2, \ldots, N_{x}-2\right\}$, then $\mathbb{D}_{\text {OBA }} \subseteq \mathbb{D}_{\text {POBA. }}$. That is, for every $\mathbf{m}=\left(m_{x}, m_{y}\right) \in \mathbb{D}_{\mathrm{OBA}}$, there exists at least one sensor pair $\left(\mathbf{n}_{1}, \mathbf{n}_{2}\right) \in \mathbb{S}_{\text {POBA }}^{2}$ such that $\mathbf{n}_{1}-\mathbf{n}_{2}=\mathbf{m}$. Note that we only need to check half of the elements in $\mathbb{D}_{\text {OBA }}$, since weight functions are symmetric, i.e., $w(\mathbf{m})=w(-\mathbf{m})$ [8]. If $\left\{\mathrm{g}_{1}, N_{x}-1-\mathrm{g}_{2}\right\}$ is a partition of $\left\{1,2, \ldots, N_{x}-2\right\}$, then $\left\{\mathrm{g}_{2}, N_{x}-1-\mathrm{g}_{1}\right\}$ is also a partition of $\left\{1,2, \ldots, N_{x}-2\right\}$. Due to this property, we can identify at least one $\left(\mathbf{n}_{1}, \mathbf{n}_{2}\right)$ pair for any given difference $\left(m_{x}, m_{y}\right)$, as listed in Table I, which proves the sufficiency.

(Necessity) If $\left\{\mathrm{g}_{1}, N_{x}-1-\mathrm{g}_{2}\right\}$ is not a partition of $\left\{1,2, \ldots, N_{x}-2\right\}$, then $g_{1} \cup\left(N_{x}-1-\mathrm{g}_{2}\right) \neq\left\{1,2, \ldots, N_{x}-2\right\}$ or $\mathrm{g}_{1}$ and $N_{x}-1-\mathrm{g}_{2}$ are not disjoint. Here we divide into two cases: For the first case, if $\mathrm{g}_{1} \cup\left(N_{x}-1-\mathrm{g}_{2}\right) \neq$ $\left\{1,2, \ldots, N_{x}-2\right\}$, there must exist $n_{0} \in\left\{1,2, \ldots, N_{x}-2\right\}$ such that $n_{0} \notin \mathrm{g}_{1}$ and $n_{0} \notin N_{x}-1-\mathrm{g}_{2}$, since $\mathrm{g}_{1}$ and $\mathrm{g}_{2}$ are subsets of $\left\{1,2, \ldots, N_{x}-2\right\}$ (the first item in Definition $4)$. We will show that, $\left(N_{x}-1-n_{0}, 1\right) \notin \mathbb{D}_{\text {POBA }}$.

Suppose there exist $\left(\mathbf{n}_{1}, \mathbf{n}_{2}\right) \in \mathbb{S}_{\text {POBA }}^{2}$ such that $\mathbf{n}_{1}-\mathbf{n}_{2}=$ $\left(N_{x}-1-n_{0}, 1\right)$. This means the $y$ coordinates of $\mathbf{n}_{1}$ and $\mathbf{n}_{2}$ must differ by 1 . According to Definition 4 , there are only two cases of $\mathbf{n}_{1}$ and $\mathbf{n}_{2}$ :

1) If $\mathbf{n}_{1} \in \mathbb{H}_{2}$ and $\mathbf{n}_{2} \in \mathbb{G}_{1}$, then the difference $\left(N_{x}-\right.$ $\left.1-n_{0}, 1\right)$ is achieved only when $\mathbf{n}_{1}=\left(N_{x}-1,1\right)$ and $\mathbf{n}_{2}=\left(n_{0}, 0\right)$. We have $\mathbf{n}_{1} \in \mathbb{H}_{2}$ but $\mathbf{n}_{2} \notin \mathbb{G}_{1}$, since $n_{0} \notin g_{1}$.

2) If $\mathbf{n}_{1} \in \mathbb{G}_{2}$ and $\mathbf{n}_{2} \in \mathbb{H}_{1}$, then $\mathbf{n}_{1}=\left(N_{x}-1-n_{0}, N_{y}-1\right)$ and $\mathbf{n}_{2}\left(0, N_{y}-2\right)$. We obtain $\mathbf{n}_{1} \notin \mathbb{G}_{2}$ since $n_{0} \notin N_{x}-$ $1-\mathrm{g}_{2}$.

For the second case, if $g_{1}$ and $N_{x}-1-g_{2}$ are not disjoint, then the size of $g_{1} \cup\left(N_{x}-1-g_{2}\right)$ can be expressed as

$$
\begin{aligned}
& \left|g_{1}\right|+\left|N_{x}-1-g_{2}\right|-\left|g_{1} \cap\left(N_{x}-1-g_{2}\right)\right| \\
& <\left|g_{1}\right|+\left|g_{2}\right|=N_{x}-2,
\end{aligned}
$$

which also implies $g_{1} \cup\left(N_{x}-1-g_{2}\right) \neq\left\{1,2, \ldots, N_{x}-2\right\}$. These arguments complete the proof.

Let us consider some examples of Theorem 1. Open box arrays are special cases of partially open box arrays with $\mathrm{g}_{1}=\left\{1,2, \ldots, N_{x}-2\right\}$ and $\mathrm{g}_{2}$ being the empty set, which satisfy Theorem 1. For the partially open box arrays in Fig. 2, the corresponding $g_{1}$ and $g_{2}$ also satisfy Theorem 1 , so their difference coarrays are hole-free, and the same as $\mathbb{D}_{\text {OBA }}$.

Furthermore, Theorem 1 offers simple and straightforward design methods for partially open box arrays with hole-free difference coarrays. The first step is to choose $g_{1}$ to be a subset of $\left\{1,2, \ldots, N_{x}-2\right\}$. Next, $g_{2}$ can be uniquely determined since $\left\{\mathrm{g}_{1}, N_{x}-1-\mathrm{g}_{2}\right\}$ is a partition of $\left\{1,2, \ldots, N_{x}-2\right\}$. Finally, the closed-form sensor locations are given in Definition 4 . The freedom in the choice of such $g_{1}$ can be exploited to reduce mutual coupling effects as explained next.

\section{B. Half Open Box Arrays}

In this subsection, we will study the half open box array, which is the partially open box array with reduced mutual coupling. This is done by setting $g_{1}$ and $g_{2}$ to be ULA with separation 2 , so that the weight function $w(1,0)$ is as small as 2 . The half open box arrays are defined as:

Definition 5 (Half open box arrays). The half open box array with parameters $N_{x}$ and $N_{y}$ is a partially open box array with

$$
\begin{aligned}
& \mathrm{g}_{1}=\left\{1+2 \ell \mid 0 \leq \ell \leq\left\lfloor\left(N_{x}-3\right) / 2\right\rfloor\right\}, \\
& \mathrm{g}_{2}=\left\{N_{x}-1-2 \ell \mid 1 \leq \ell \leq\left\lfloor\left(N_{x}-2\right) / 2\right\rfloor\right\} .
\end{aligned}
$$

According to (7), $g_{1}$ represents an ULA whose left-most element is 1 and the interelement spacing is 2 . It can be shown that (7) and (8) meet Theorem 1, so that the difference coarray of the half open box array is the same as that of the open box array, and hence, hole-free. The sensor positions for the half open box array can also be obtained from Defintion 4 and 5 readily, even for large $N_{x}$ and $N_{y}$.

Fig. 2(b) illustrates the half open box array with $N_{x}=18$ and $N_{y}=10$. It can be seen that, $\left|\mathrm{g}_{1}\right|=\left|\mathrm{g}_{2}\right|=8$ and the weight functions for Fig. 2(b) are listed as follows:

$$
w(1,0)=2, w(0,1)=18, w(1,1)=1, w(1,-1)=1 .
$$

Compared to the open box array in Fig. 1(d), w(1,0) decreases from 17 to 2 while $w(0,1), w(1,1)$, and $w(1,-1)$ remain the same. To be more precise, the weight function $w(1,0)$ can be specified by the following Theorem:

Theorem 2. If $N_{x} \geq 3$, then the weight function $w(1,0)$ for the half open box array is 2.

Proof. To evaluate $w(1,0)$, it suffices to consider the elements whose $y$ coordinates are either 0 or $N_{y}-1$, due to Definition 4. Since $N_{x} \geq 3, \mathrm{~g}_{1}$ is not empty. It is obvious that the elements $(1,0)$ and $(0,0)$ lead to $w(1,0)$. First consider $N_{x}$ to be an odd number. According to (7), $N_{x}-2 \in \mathrm{g}_{1}$, so $\left(N_{x}-1,0\right)$ and $\left(N_{x}-2,0\right)$ also contribute to $w(1,0)$. In this case, the smallest and the largest elements in $\mathrm{g}_{2}$ are 2 and $N_{x}-3$, respectively, implying there are no sensor pairs with separation 1 if the $y$ coordinates are $N_{y}-1$. On the other hand, if $N_{x}$ is even, the only two sensor pairs contributing to $w(1,0)$ are $(1,0),(0,0)$ and $\left(1, N_{y}-1\right),\left(0, N_{y}-1\right)$, which completes this proof.

Therefore, the estimation performance for the half open box array would be better than the open box array in the presence of mutual coupling, as we shall demonstrate next.

\section{NUMERICAL EXAMPLES}

Consider the planar arrays with 36 sensors, listed in Fig. 1 and 2. We choose $D=10$, uncorrelated, equal-power sources, whose normalized DOAs are randomly drawn from $\left(\bar{\theta}_{i}, \bar{\phi}_{i}\right) \in[-0.45,0.45]^{2}$. The SNR is $0 \mathrm{~dB}$ and the number 
TABLE I

12 CASES IN THE PRoOF OF THEOREM 1

\begin{tabular}{|c|c|c|c|c|}
\hline Case & $m_{x}$ & $m_{y}$ & $\mathbf{n}_{1}$ & $\mathbf{n}_{2}$ \\
\hline $\begin{array}{l}1 \\
2 \\
3 \\
4\end{array}$ & $\begin{array}{c}0 \\
\mathrm{~g}_{1} \\
N_{x}-1-\mathrm{g}_{2} \\
N_{x}-1\end{array}$ & 0 & $\begin{array}{c}(0,0) \in \mathbb{S}_{\mathrm{POBA}} \\
\left(m_{x}, 0\right) \in \mathbb{G}_{1} \\
\left(N_{x}-1, N_{y}-1\right) \in \mathbb{S}_{\mathrm{POBA}} \\
\left(N_{x}-1,0\right) \in \mathbb{S}_{\mathrm{POBA}}\end{array}$ & $\begin{aligned} &(0,0) \in \mathbb{S}_{\mathrm{POBA}} \\
&(0,0) \in \mathbb{S}_{\mathrm{POBA}} \\
&\left(N_{x}-1-m_{x}, N_{y}-1\right) \in \mathbb{G}_{2} \\
&(0,0) \in \mathbb{S}_{\text {POBA }}\end{aligned}$ \\
\hline $\begin{array}{l}5 \\
6 \\
7 \\
8\end{array}$ & $\begin{array}{c}0 \\
N_{x}-1-\mathrm{g}_{1} \\
\mathrm{~g}_{x}-1\end{array}$ & $1, \ldots, N_{y}-1$ & $\begin{array}{c}\left(0, m_{y}\right) \in \mathbb{S}_{\mathrm{POBA}} \\
\left(N_{x}-1, m_{y}\right) \in \mathbb{S}_{\mathrm{POBA}} \\
\left(m_{x}, N_{y}-1\right) \in \mathbb{G}_{2} \\
\left(N_{x}-1, m_{y}\right) \in \mathbb{S}_{\mathrm{POBA}}\end{array}$ & $\begin{array}{c}(0,0) \in \mathbb{S}_{\mathrm{POBA}} \\
\left(N_{x}-1-m_{x}, 0\right) \in \mathbb{G}_{1} \\
\left(0, N_{y}-1-m_{y}\right) \in \mathbb{S}_{\mathrm{POBA}} \\
(0,0) \in \mathbb{S}_{\mathrm{POBA}}\end{array}$ \\
\hline $\begin{array}{c}9 \\
10 \\
11 \\
12 \\
\end{array}$ & $\begin{array}{c}0 \\
\mathrm{~g}_{1} \\
N_{x}-1-\mathrm{g}_{2} \\
N_{x}-1 \\
\end{array}$ & $-N_{y}+1, \ldots,-1$ & $\begin{array}{c}(0,0) \in \mathbb{S}_{\mathrm{POBA}} \\
\left(m_{x}, 0\right) \in \mathbb{G}_{1} \\
\left(N_{x}-1, N_{y}-1+m_{y}\right) \in \mathbb{S}_{\text {POBA }} \\
\left(N_{x}-1, N_{y}-1+m_{y}\right) \in \mathbb{S}_{\text {POBA }} \\
\end{array}$ & $\begin{aligned} &\left(0,-m_{y}\right) \in \mathbb{S}_{\text {POBA }} \\
&\left(0,-m_{y}\right) \in \mathbb{S}_{\text {POBA }} \\
&\left(N_{x}-1-m_{x}, N_{y}-1\right) \in \mathbb{G}_{2} \\
&\left(0, N_{y}-1\right) \in \mathbb{S}_{\text {POBA }} \\
&\end{aligned}$ \\
\hline
\end{tabular}
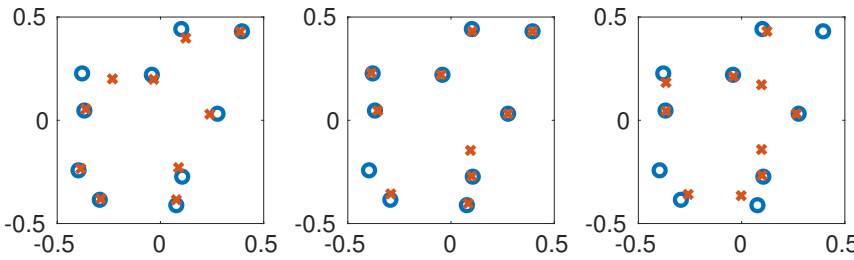

(a) $E=0.054289$

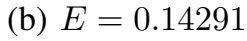

(c) $E=0.17525$
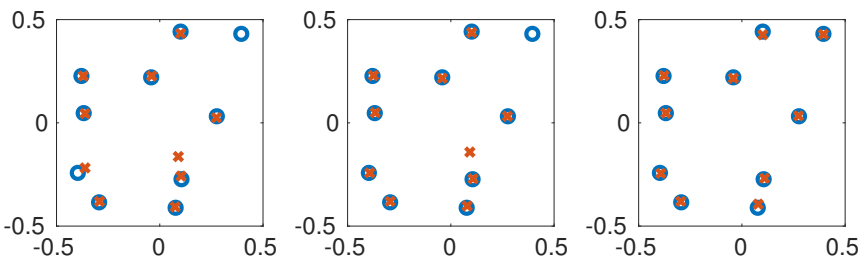

(d) $E=0.15976$

(f) $E=0.0078139$
Fig. 3. The true source directions (in circles) and the estimated directions (in crosses) for (a) the URA, (b) the billboard array, (c) the 2D nested array, (d) the open box array, (e) the partially open box array, and (f) the half open box array. The horizontal and vertical axes stand for $\bar{\theta}$ and $\bar{\phi}$, respectively. The error is defined as $E=\left(\sum_{i=1}^{D}\left(\left(\bar{\theta}_{i}-\widehat{\bar{\theta}}_{i}\right)^{2}+\left(\bar{\phi}_{i}-\widehat{\bar{\phi}}_{i}\right)^{2}\right) / D\right)^{1 / 2}$, where $\left(\bar{\theta}_{i}, \bar{\phi}_{i}\right)$ and $\left(\widehat{\bar{\theta}}_{i}, \widehat{\bar{\phi}}_{i}\right)$ are the true and estimated normalized DOA, respectively.

of snapshots is 500. The mutual coupling model (5) has $c(1)=0.25, c(\ell)=c(1) e^{-j 2 \pi(\ell-1) / 5} / \ell$, and $B=10$. The data is generated using (4) and the DOA are estimated from 2D unitary ESPRIT [18] on the finite-snapshot version of (3). The results are shown in Fig. 3, where the true directions and the estimated directions are marked in circles and crosses, respectively. In this example, only the newly proposed half open box array resolves all these sources correctly, while the others miss at least one source. Note that we do not apply any decoupling algorithm to compensate mutual coupling. The performance improvement is due to the array geometry.

Note that the number of sources is much smaller than sensors $(10 \ll 36)$. Theoretically, sparse arrays can resolve more sources than sensors in the absence of mutual coupling [15]. However, if mutual coupling is present, this is more challenging, and it will be explored in greater detail in future.

\section{CONCLUding REMARKS}

In this paper, we first proposed partially open box arrays, which generalize open box arrays by redistributing some sensors while preserving the difference coarray. We then developed half open box arrays which are partially open box arrays with the additional property that mutual coupling effects are reduced significantly.

It can be seen from (9) that the weight function $w(0,1)$ is still large, compared to $w(1,0), w(1,1)$, and $w(1,-1)$, since the elements whose $x$ coordinates are 0 or $N_{x}-1$ are fixed. In the future, it will be of considerable interst to decrease $w(0,1)$ by relocating those sensors, so that mutual coupling can be reduced further.

\section{REFERENCES}

[1] H. L. Van Trees, Optimum Array Processing: Part IV of Detection Estimation, and Modulation Theory. Wiley Interscience, 2002.

[2] M. I. Skolnik, Introduction to Radar Systems, 3rd ed. McGraw Hill, 2001.

[3] S. Haykin, Array Signal Processing. Prentice-Hall, 1984.

[4] B. Friedlander and A. Weiss, "Direction finding in the presence of mutual coupling," IEEE Trans. Antennas Propag., vol. 39, no. 3, pp. 273-284, Mar 1991.

[5] C. A. Balanis, Antenna Theory: Analysis and Design, 4th ed. John Wiley \& Sons, 2016.

[6] C.-L. Liu and P. P. Vaidyanathan, "Super nested arrays: Linear sparse arrays with reduced mutual coupling - Part I: Fundamentals," to appear in IEEE Trans. Signal Proc.

[7] A. T. Moffet, "Minimum-redundancy linear arrays," IEEE Trans. Antennas Propag., vol. 16, no. 2, pp. 172-175, 1968.

[8] P. Pal and P. P. Vaidyanathan, "Nested arrays: A novel approach to array processing with enhanced degrees of freedom," IEEE Trans. Signal Proc., vol. 58, no. 8, pp. 4167-4181, Aug 2010.

[9] P. P. Vaidyanathan and P. Pal, "Sparse sensing with co-prime samplers and arrays," IEEE Trans. Signal Proc., vol. 59, no. 2, pp. 573-586, Feb 2011.

[10] C.-L. Liu and P. P. Vaidyanathan, "Super nested arrays: Linear sparse arrays with reduced mutual coupling - Part II: High-order extensions," to appear in IEEE Trans. Signal Proc., 2016.

[11] S. Qin, Y. Zhang, and M. Amin, "Generalized coprime array configurations for direction-of-arrival estimation," IEEE Trans. Signal Proc., vol. 63, no. 6, pp. 1377-1390, March 2015.

[12] C. R. Greene and R. C. Wood, "Sparse array performance," J. Acoust. Soc. Am., vol. 63, no. 6, pp. 1866-1872, 1978.

[13] H. C. Pumphrey, "Design of sparse arrays in one, two, and three dimensions," J. Acoust. Soc. Am., vol. 93, no. 3, pp. 1620-1628, 1993.

[14] P. Pal and P. P. Vaidyanathan, "Nested arrays in two dimensions, Part I: Geometrical considerations," IEEE Trans. Signal Proc., vol. 60, no. 9, pp. 4694-4705, Sept 2012.

[15] pp. dimensional array processing," IEEE Trans. Signal Proc., vol. 60, no. 9 , pp. 4706-4718, Sept 2012.

[16] C.-L. Liu and P. P. Vaidyanathan, "Remarks on the spatial smoothing step in coarray MUSIC," IEEE Signal Proc. Lett., vol. 22, no. 9, pp. 1438-1442, Sept 2015.

[17] T. Jiang, N. D. Sidiropoulos, and J. M. F. ten Berge, "Almost-sure identifiability of multidimensional harmonic retrieval," IEEE Trans. Signal Proc., vol. 49, no. 9, pp. 1849-1859, Sep 2001.

[18] M. D. Zoltowski, M. Haardt, and C. P. Mathews, "Closed-form 2-D angle estimation with rectangular arrays in element space or beamspace via unitary ESPRIT," IEEE Trans. Signal Proc., vol. 44, no. 2, pp. 316328, Feb 1996. 